\title{
Impacto económico en la provincia de Tungurahua en relación con los eventos adversos
}

Fecha de recepción: 2021-07-14 • Fecha de aceptación: 2021-09-15 • Fecha de publicación: 2021-10-10

Jorge Oswaldo Tamayo Viera ${ }^{1}$ Tecnológico Superior Universitario España, Ecuador jorge.tamayo@iste.edu.ec

https://orcid.org/0000-0002-2554-8684

Vicente Leonardo Pérez Yauli² Estudio Jurídico Acosta \& Asociados, Ecuador p.leonardo94@yahoo.es https://orcid.org/0000-0003-1937-7820

Edison Manuel Arroba Freire ${ }^{3}$

Tecnológico Superior Universitario España, Ecuador edison.arroba@iste.edu.ec https://orcid.org/0000-0002-7196-0784

\section{RESUMEN}

El presente estudio de carácter investigativo tuvo por objetivo realizar un análisis sobre los principales eventos adversos y el impacto de estos en la economía dentro de la provincia de Tungurahua. Los métodos de investigación empleados fueron, cualitativo, cuantitativo, descriptivo y bibliográfico; se partió de la revisión de documentos e informes relacionados con el objeto de estudio, hasta la obtención de resultados representados en figuras con expresiones porcentuales e interpretaciones, relativos a defunciones, desempleo, incendios forestales, ayuda de organizaciones gubernamentales y asistencia técnica, uso del suelo productivo, venta de ganado, agricultura, mercado laboral y turismo, registrados en el periodo comprendido entre los años 2018 - 2020 . Cualquier evento adverso o desastre como también se los conoce tiene su origen en la naturaleza y 
sus consecuencias son negativas por las pérdidas que representan. La Constitución de la República en su texto ha previsto las medidas necesarias para fortalecer la capacidad de acción ante estos eventos, así como la toma de medidas para reducirlos. La constante incidencia de estos hechos ha motivado la expedición de planes, política, programas y proyectos con el propósito de frenar en algo su ocurrencia y sus efectos.

PALABRAS CLAVE: desastres, emergencia, crisis, riesgo, impacto.

\section{ABSTRACT}

The present study of a research was aimed at performing an analysis of the main adverse events and the impact of these in the economy within the province of Tungurahua. The methods of research employed were, qualified, descriptive and bibliographical; it was broken from the review of documents and reports related to the object of study, until obtaining results represented in figures with percentage expressions and interpretations, relating to deaths, unemployment, forest fires, government organizations and technical assistance, land use productive, sale of livestock, agriculture, labor market and tourism, registered in the period between 2018 - 2020. Any adverse event or disaster as well as known has its origin in nature and its consequences are negative for the losses they represent. The Constitution of the Republic in his text provided the necessary measures to strengthen the capacity of action in these events, and taking action to reduce them. The constant occurrence of these events has led to the issuance of plans, policies, programs and projects in order to stop something occurrence and effects. 


\section{Introducción}

Un evento adverso es definido como un fenómeno que produce efectos desfavorables en las personas, sea de forma individual o colectiva, cuyo acontecimiento produce efecto en la economía, sistemas sociales y el medio ambiente, su origen obedece a la propia naturaleza, la actividad humana o a ambas, en forma combinada, pudiendo causar crisis, emergencias o desastres (OMS, 2014, citado en Mendoza, 2019, p.6).

El término evento adverso comúnmente es empleado en el lenguaje cotidiano para referirse a los llamados desastres, que tienen incidencia en ciertas actividades de carácter económico como la agricultura, ganadería, turismo e incluso en la actividad laboral de las personas. El emplear las expresiones crisis, emergencias, desastres o catástrofes indistintamente, aparentemente en teoría es acertada, en la práctica no es así, pues las cuatro expresiones tienen marcadas diferencias en cuanto al daño que producen en la circunscripción territorial en la que acontecen, como también las consecuencias que de ellas derivan (UNESCO, 2011, p.12).

Crisis es un término utilizado comúnmente para referir al estado por el que atraviesa un pueblo o de una persona, en el caso particular con incidencia en las emociones de quien es afectada e imposibilitada de superarla por sus propios medios; estos fenómenos están relacionados estrechamente con el acontecimiento de lo que comúnmente en la jerga popular se ha denominado como desastres, en cuanto se producen, afectan a la población que la sufre en los diferentes contextos de su cotidianidad (UNESCO, 2011, p.12).

Una emergencia producida por un desastre o amenaza afecta a una sociedad en cuanto corta el desarrollo sus actividades habituales; por su acaecimiento se ven perjudicadas a más de la vida humana, la salud, bienes y servicios, como también la naturaleza y los ecosistemas existentes; si bien estas situaciones pueden ser manejadas de acuerdo a la capacidad de respuesta de la población, de los organismos gubernamentales y técnicos, esto depende también en gran parte del grado de preparación (Hernández, 2020).

Por otra parte, desastre se entiende como el acontecimiento inevitable que se produce en una sociedad, afectando de forma directa sus actividades, propias con grandes y graves repercusiones, entre estas, muerte de personas, perdidas económicas, materiales y del medio ambiente, consecuencias que, por la gravedad, difícilmente pueden ser superadas, ya que en mucho aventajan la capacidad de respuesta y la preparación de la colectividad (UNISDR, 2002, citado en UNESCO, 2014, p. 8).

En la actualidad, uno de los mayores factores de riesgo para el Ecuador y las provincias que lo componen representan los volcanes que se encuentran en actividad, más aún aquellos ubicados cerca de zonas donde se encuentran asentamientos humanos, dedicados a las labores del agro (agricultura, ganadería); cercanía que ha sido determinante en la ocurrencia de fenómenos naturales numerosos y graves, que han quedado registrados para la historia y particularmente en la memoria de los pobladores. Uno de estos hechos se produjo en 1999 con la erupción del volcán Pichincha con grandes cantidades de ceniza volcánica, que para la época evidenció la falta de 
preparación y conocimiento técnico para hacer frente a este tipo de sucesos (Yánez Lucero, 2017, p.1).

De acuerdo a los estudios comparativos realizados entre Ecuador y Perú, países colindantes, en relación a la actividad sísmica que se produce en estas localidades, los datos de modo teórico deberían ser similares; sin embargo, en la realidad no ocurre así, en tanto que en suelo peruano la actividad sísmica se mantiene estable en el lado ecuatoriano, existe gran diferencia, pues, dicha actividad se presenta de forma escalonada con épocas es menor y en otras de mayor escala; fenómeno que en geología es conocido como reactivación sísmica, y; que de una u otra manera, influye en el normal desarrollo de la sociedad (Moncayo et al., 2017).

La ocurrencia de eventos adversos o desastres en Ecuador ha estado marcada por la reactivación de sus volcanes como el Tungurahua, Cotopaxi, recientemente el Sangay en la provincia de Morona Santiago, también por el fenómeno del Niño en la región Litoral, caracterizado por la presencia de lluvias e inundaciones, deslizamientos de tierra e incendios, ocasionando grandes pérdidas para el país. Sin embargo, el mayor desastre que ha tenido que afrontar Ecuador, y que ha quedado registrado en su historia, es el terremoto de abril de 2016 en la costa ecuatoriana con una escala de 7.8; suceso que ocasionó pérdidas humanas, materiales y económicas. Actualmente este hecho ha sido superado por el acontecimiento de mayor transcendencia a nivel mundial, la pandemia del Covid - 19, que ha generado a nivel local y en las diferentes latitudes del mundo, crisis de índole económica, política y social (Maguiña Vargas et al., 2020).

Dentro del territorio nacional, el volcán Tungurahua, dada su actividad, es considerado como uno de los que representa mayores riesgos, como consecuencias de su efecto eruptivo en las localidades aledañas se han producido sismos, deslizamientos de tierra, pérdidas de cultivos y de ganado producto de la ceniza volcánica, incluso la pérdida de vidas humanas. Debido a su constante actividad y a las consecuencias negativas, los entes gubernamentales respaldados en la normativa constitucional vigente, y a través de los Gobiernos autónomos descentralizados han trabajado en la generación de medidas que coadyuven a prevenir sus efectos adversos y a mejorar la capacidad de respuesta de manera particular de los habitantes cercanos al volcán.

El fenómeno del Niño, conforme se ha señalado con anterioridad, ha traído consecuencias negativas para las poblaciones que a lo largo de los años se han visto afectadas. En la región Sierra se produce un fenómeno contrario caracterizado por las sequias y altas temperaturas "los incendios forestales"; pero que de manera similar a la presencia de fuertes lluvias trae consigo consecuencias negativas como erosión de suelos cultivables, perdida de grandes extensiones de bosques, perdida de tierras de pastoreo de ganado, impactando de forma decisiva en el Producto Interno Bruto (PIB) del país, ocasionando su disminución, que a su vez desencadena una serie de efectos que causan sobresalto en la sociedad (Villavicencio Silva, 2019).

En los últimos años, las áreas agrestes de la provincia de Tungurahua se han visto mermadas como efecto de la presencia de incendios forestales, de acuerdo a los datos oficiales, las perdidas bordean las ochocientas hectáreas de bosques y tierras de cultivo. Con el propósito de frenar estos hechos, el legislador ha considerado pertinente integrar a la legislación penal ecuatoriana sanciones para quienes ocasionen este tipo de desastres, así, el artículo 246 del Código Orgánico 
Integral Penal (Asamblea Nacional del Ecuador, 2014), sanciona con privación de la libertad a la persona que por cuenta propia o por intermedio de otra ocasione incendios en las áreas verdes protegidas en el territorio del Ecuador (Gestión de Riesgos y Emergencias, 2018, citado en Paredes \& Sánchez, 2020, p.3).

A lo largo de la historia de la humanidad ésta ha tenido que enfrentar un sinnúmero de eventos adversos o desastres (inundaciones, erupciones volcánicas, incendios, sismos, etc.), sin lugar a duda, cada uno de estos eventos han ocasionado daño y destrucción en el ambiente y sus ecosistemas y, por ende, a las condiciones de vida del ser humano. Actualmente, y de acuerdo a lo prescrito en la Constitución de la República del Ecuador (2008), a nivel interno, el Estado ecuatoriano considera como políticas de gestión de riesgo los derechos y protección de la naturaleza, la participación y seguridad de la población, buscando como medida prioritaria fortalecer en la ciudadanía y en las entidades públicas y privadas capacidades para identificar los riesgos inherentes a sus respectivos ámbitos de acción, informar sobre ellos, e incorporar acciones tendientes a reducirlos.

El Estado, a través de las instituciones públicas que lo integran han dispuesto en la parte de su contingente económico, material, logístico, humano; para la toma de las mejores decisiones, la formulación y expedición de planes, políticas y programas, que vengan a disminuir las probabilidades de ocurrencia de desastres, y; en caso inevitable mitigar sus consecuencias negativas. Esto implica desarrollar procesos de planificación colaborativos y participativos con actores gubernamentales y sociales, con el propósito de prevenir o responder mejor, según sea el caso a una situación de emergencia o desastre.

De lo dicho, previa a la ocurrencia y en el supuesto una vez producido un evento adverso se activan operativos de emergencia a través de la participación de organismos técnicos constituidos y adscritos al servicio integrado de seguridad como el Comité de Operaciones de Emergencia (COE), ECU 911, la Secretaría de Gestión de Riesgos, Cuerpo de bomberos, Policía Nacional y la Dirección de Tránsito.

Conforme con el Proyecto de Ley del Sistema Nacional Descentralizado de Gestión de Riesgos (2013) y el principio de descentralización, la gestión de riesgos corresponde a cada Gobierno autónomo descentralizado (GAD) dentro del ámbito de sus competencias, de ahí la conformación de los Centros de Operaciones de Emergencia con jurisdicción cantonal y parroquial en estrecha relación con el COE nacional, organismos que tienen a su cargo planificar y gestionar los planes, proyectos y programas a fin de dar respuesta oportuna e inmediata a los diferentes eventos adversos que se susciten en las suscripciones con sujeción a su autoridad.

En el territorio nacional en cada provincia que lo conforma en diferentes tiempos han afrontados eventos adversos y sus efectos; siendo la población considerada como lumpen proletariado, mayormente afectada dada sus condiciones de vida y pobreza, misma que no disponen de los medios o capacidad necesaria para sobreponerse ante una o varias situaciones inciertas o de emergencia. 
En este orden, es indispensable que el propio Estado y la sociedad encuentren un punto de equilibrio para desarrollar y fomentar capacidades de adaptabilidad, en el afán de dar respuesta eficiente y rápida ante cualquier evento adverso, contando para ello con procesos de planificación adecuados que identifiquen las principales amenazas, zonas de mayor vulnerabilidad, minimizando de este modo el impacto negativo que pudiera producirse en la sociedad (Gaibor \& Pilco, 2021).

\section{Metodología}

En el desarrollo de la presente investigación se emplearon los métodos cualitativo y cuantitativo, descriptivo y bibliográfico, el punto de partida refirió al análisis de documentos con contenido relativo a eventos adversos, desastres, emergencias; como también informes del Instituto Nacional de Estadísticas y Censos (INEC), relacionados a defunciones, desempleo, incendios forestales, ayuda de organizaciones gubernamentales y asistencia técnica, uso del suelo productivo, venta de ganado, agricultura, mercado laboral y turismo.

Estos indicadores han sido representados mediante figuras y tablas estadísticas, permitiendo su interpretación y comprensión, en cuanto tiene que ver con la ocurrencia de los denominados eventos adversos y su incidencia o impacto en la economía en la provincia de Tungurahua.

\section{Resultados}

\section{Figura 1}

Número de defunciones

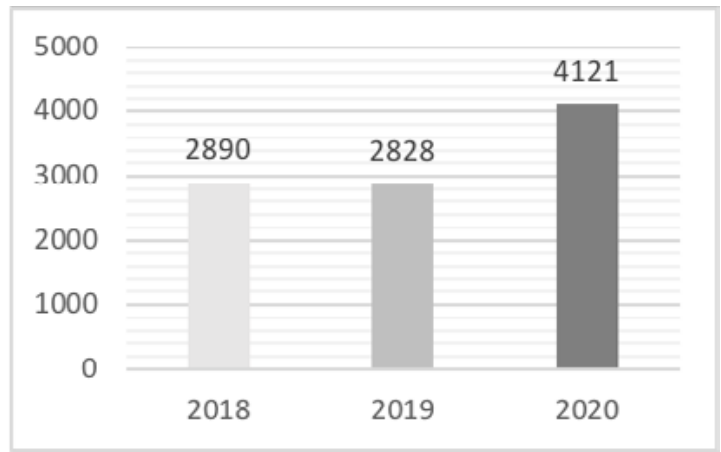

Fuente: Registro Estadístico de Defunciones Generales (2021)

En Tungurahua se registró un incremento de defunciones, esto se debe a que en el último año se produjo un evento adverso mundial por la nueva enfermedad denominada pandemia Covid 2019, en el año 2018 se contabilizan un número de dos mil ochocientas noventa personas fallecidas, para el año 2019 se registran dos mil ochocientas veinte y ocho defunciones, para posteriormente en el año 2020 tener un incremento elevado del $45,7 \%$ con cuatro mil ciento veinte y un personas fallecidas en relación al año 2019, lo cual ocasionó también gastos imprevistos por sus decesos. 


\section{Figura 2}

Tasa de desempleo

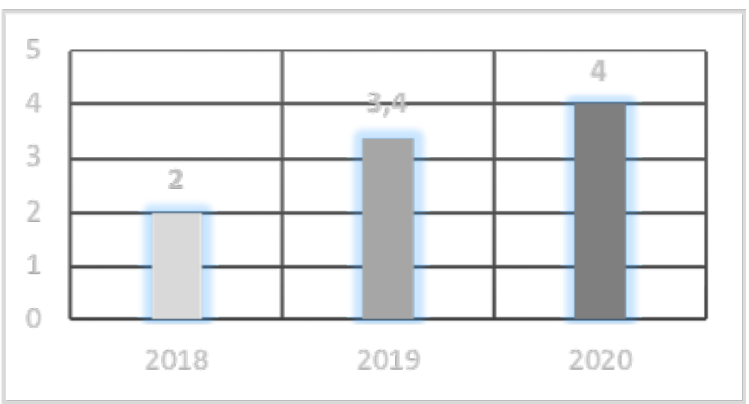

Fuente: Encuesta Nacional de Empleo, Desempleo, y Subempleo (2021)

En Tungurahua se presenta un alto índice de desempleo, registrándose una tasa del $2 \%$ para el año 2018, para el año 2019 se incrementa un 1,4\% en relación al año anterior, lo cual se manifiesta en la reducción de la fuerza laboral del sector de pequeñas y medianas empresas. El año 2020 se desencadena un cierre de negocios y un despido masivo, lo cual duplica la tasa a un $4 \%$ tomando como año de referencia el 2018 , debido al confinamiento de la pandemia que afecta la dinámica económica en Tungurahua. Cabe recalcar que no se encuentra registrado los subempleos y empleos informales en la provincia.

\section{Figura 3}

Incendios forestales

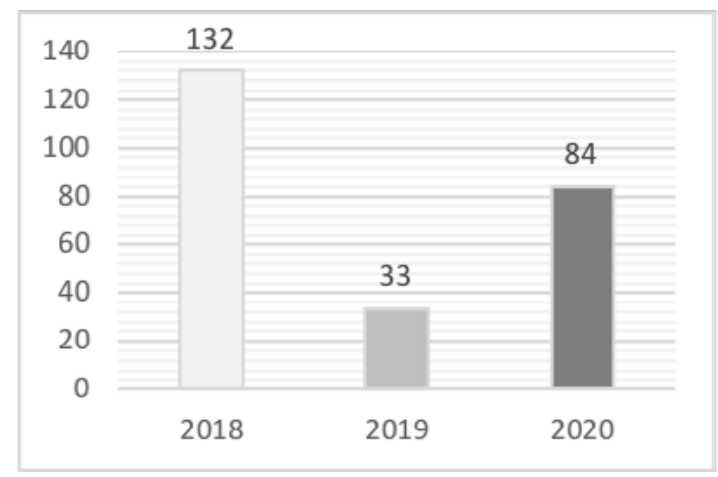

Fuente: Servicio Nacional de Gestión de Riesgos y Emergencias (2020)

En la provincia de Tungurahua se registraron ciento treinta y dos incendios forestales para el año 2018, en el año 2019 bajó el número de incendios forestales a treinta y tres, finalmente en el año 2020 se incrementa en el 154,55\%, equivalente a ochenta y cuatro eventos de incendios forestales, producido por la quema de árboles de personas que atentan contra los recursos naturales, generando pérdidas económicas en los sembríos. 


\section{Tabla 1}

Ayuda recibida durante la pandemia en la provincia de Tungurahua

\begin{tabular}{lll}
\hline Dificultad presentada & SI & NO \\
\hline $\begin{array}{l}\text { Ministerio de Agricultura y } \\
\text { Ganadería }\end{array}$ & $0,44 \%$ & $99,56 \%$ \\
\hline $\begin{array}{l}\text { Secretaria de Gestión de } \\
\text { Riesgos }\end{array}$ & $0,25 \%$ & $99,75 \%$ \\
\hline Gobierno Central & $0,78 \%$ & $99,22 \%$ \\
\hline $\begin{array}{l}\text { Gobierno Autónomo } \\
\text { Descentralizado Municipal }\end{array}$ & $2,02 \%$ & $97,98 \%$ \\
\hline $\begin{array}{l}\text { Gobierno Autónomo } \\
\text { Descentralizado Provincial }\end{array}$ & $0,69 \%$ & $99,31 \%$ \\
\hline Organización no Gubernamental & $0,29 \%$ & $99,71 \%$ \\
\hline Otro & $0,93 \%$ & $99,07 \%$ \\
\hline
\end{tabular}

Fuente: Instituto Nacional de Estadísticas y Censos (2021)

A su vez, se registra $99,07 \%$ de ayuda recibida en la provincia de Tungurahua por parte de las organizaciones gubernamentales, llamadas a brindar el apoyo correspondiente durante la pandemia, la logística y los recursos desplegados por el Gobierno central son limitados frente a la magnitud de este evento adverso. La falta de asistencia técnica se evidencia con un $99,07 \%$ en todo el sector.

\section{Figura 4}

Asistencia técnica durante la pandemia

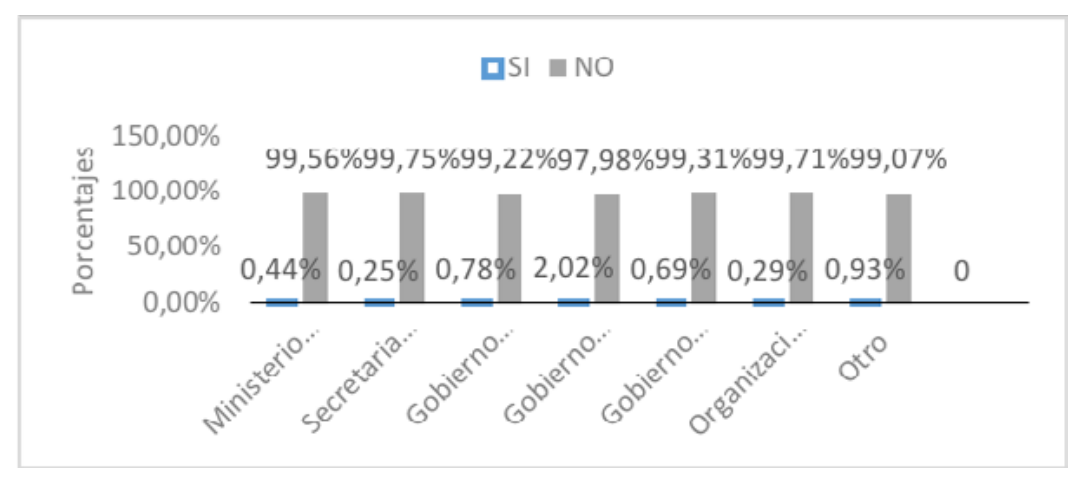

Fuente: Instituto Nacional de Estadísticas y Censos (2019)

No se refleja ayuda recibida o asistencia técnica registrada en el año 2020 por parte de las diferentes entidades gubernamentales en la provincia de Tungurahua, motivo por el cual no llega al $1,00 \%$ de su contribución. El Gobierno central presenta un $0,78 \%$ de asistencia, el Gobierno autónomo descentralizado municipal contabiliza un 2,02\% de ayuda en la provincia, otras instituciones suman un $0,93 \%$ de aporte para mitigar en parte la pandemia Covid-19. 


\section{Figura 5}

Uso del suelo

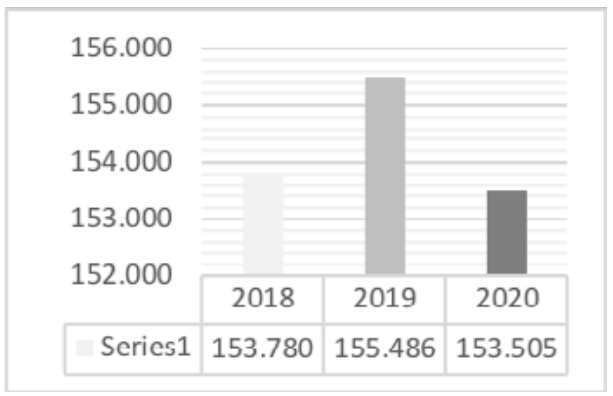

Fuente: Instituto Nacional de Estadísticas y Censos

El uso del suelo agrícola en el año 2018 fue de ciento cincuenta y tres mil setecientos ochenta hectáreas; mientras que en el 2019 existe un aumento notable de la extensión de la frontera agrícola a ciento cincuenta y cinco mil cuatrocientos ochenta y seis hectáreas, lo cual afecta directamente a los suelos del páramo por el uso indiscriminado de agroquímicos, ocasionado la perdida especies de plantas nativas; finalmente para el año 2020 la frontera agrícola ha disminuido en $1,27 \%$ de su capacidad agrícola productiva.

\section{Figura 6}

Venta ganado vacuno machos

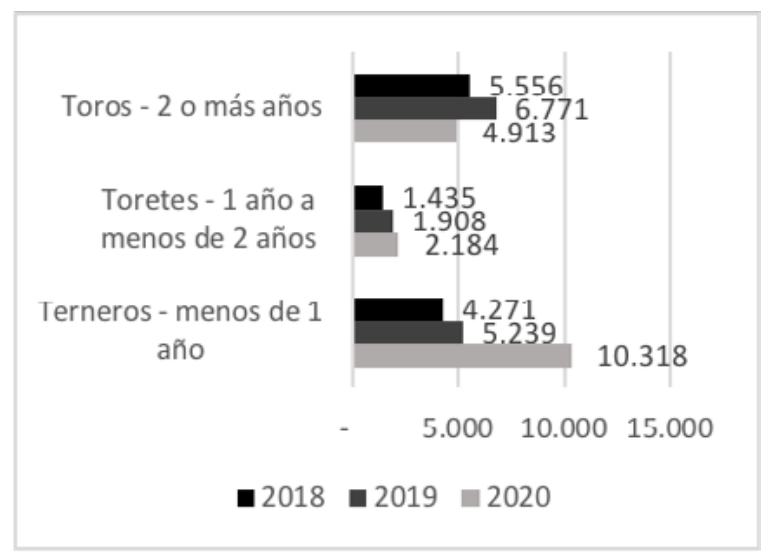

Fuente: Instituto Nacional de Estadísticas y Censos (2020)

Durante el periodo del 2018 al 2020, la venta de ganado vacuna machos de dos o más años de edad registró un valor de cuatro mil novecientas trece unidades, el confinamiento por la pandemia Covid-19 afectó la comercialización normal y correspondiente venta de toros. En lo que tiene que ver a los toretes y terneros no se registraron mayores inconvenientes en el proceso de la crianza del ganado inferior a dos años de edad. 


\section{Figura 7}

Venta ganado vacuno hembras

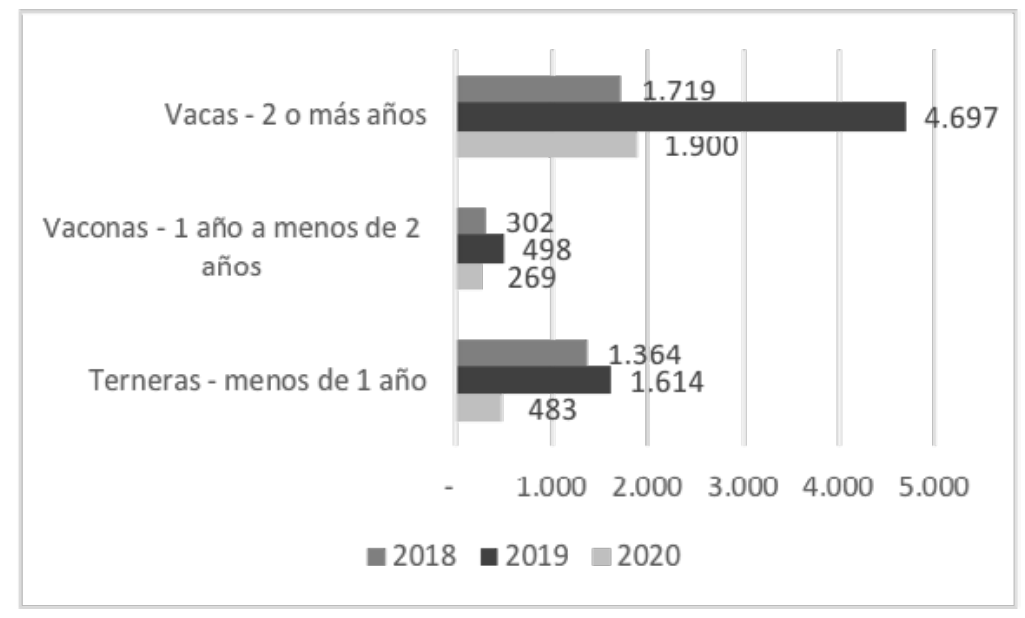

Fuente: Instituto Nacional de Estadísticas y Censos (2020)

Se registró una disminución del 70,07\% en el número de unidades vendidas de ganado vacuno hembras de menos de un año de edad para el año 2020, registrándose ventas de cuatrocientas ochenta y tres terneras, lo cual representa la tercera parte de las ventas obtenidas en los años 2018 y 2019. De igual manera se contabiliza una disminución del 45,98\% en las ventas del ganado hembra, de un año a menos de dos años de edad. Se reporta una baja del 59,55\% en la venta de las vacas en la provincia de Tungurahua.

\section{Figura 8}

Venta de ganado otras especies

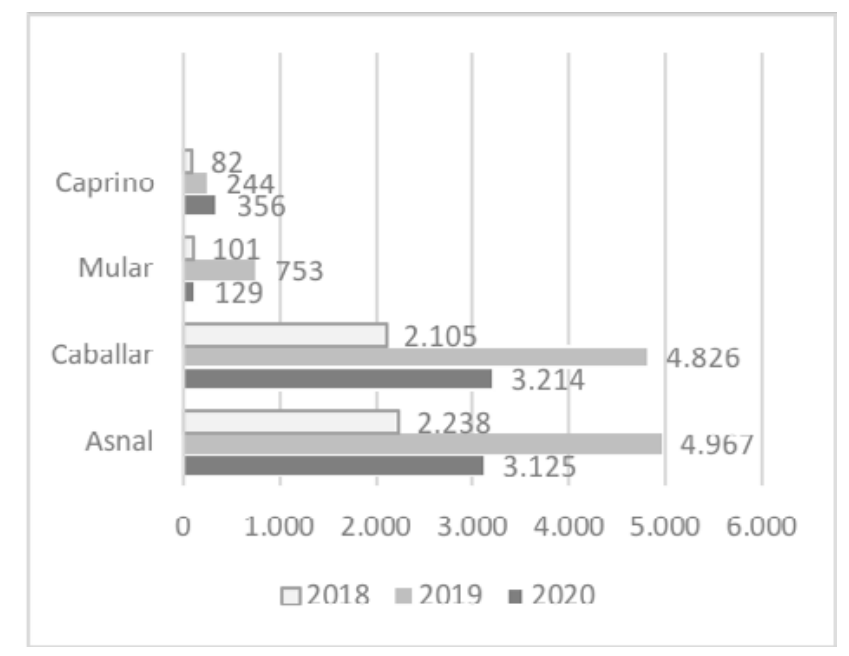

Fuente: Instituto Nacional de Estadísticas y Censos (2020) 
Se puede apreciar que, en el año 2020, se ha registrado una disminución en la venta de ganado otras especies como el mular que apenas se han vendido ciento veinte y nueve especies que representa un decremento del $82,87 \%$, el ganado caballar registra una baja del $33,40 \%$ y el ganado asnal con ventas registradas de tres mil ciento veinte y cinco unidades lo cual evidencia una disminución del $37,09 \%$ en relación a sus ventas del año 2019, debido a los eventos adversos registrados.

Figura 9

Personas productoras sin remuneración

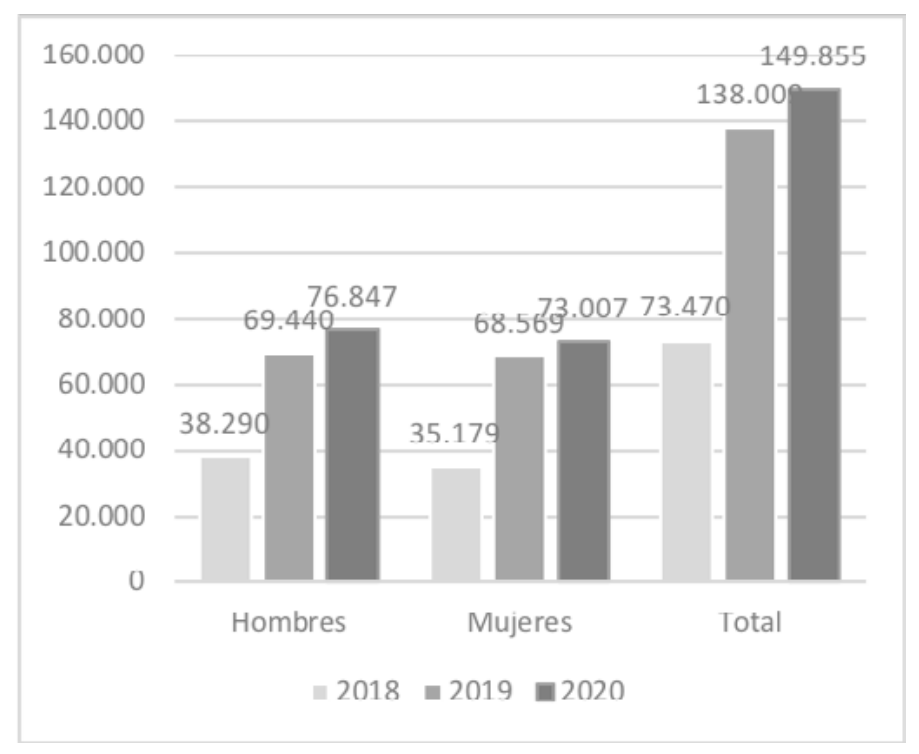

Fuente: Instituto Nacional de Estadísticas y Censos (2021)

Existen un número considerable de personas y familias productoras en el campo que se encuentran sin relación de dependencia de institución pública o privada, evidenciándose un parcial incremento de mano de obra para el año 2020 del 8,58\%, en relación al incremento significativo del año 2019 del 87,84\% en base al año 2018 que se contrataron casi la mitad de personas en el sector agrícola en la provincia de Tungurahua. 


\section{Figura 10}

Trabajadores remunerados permanentes

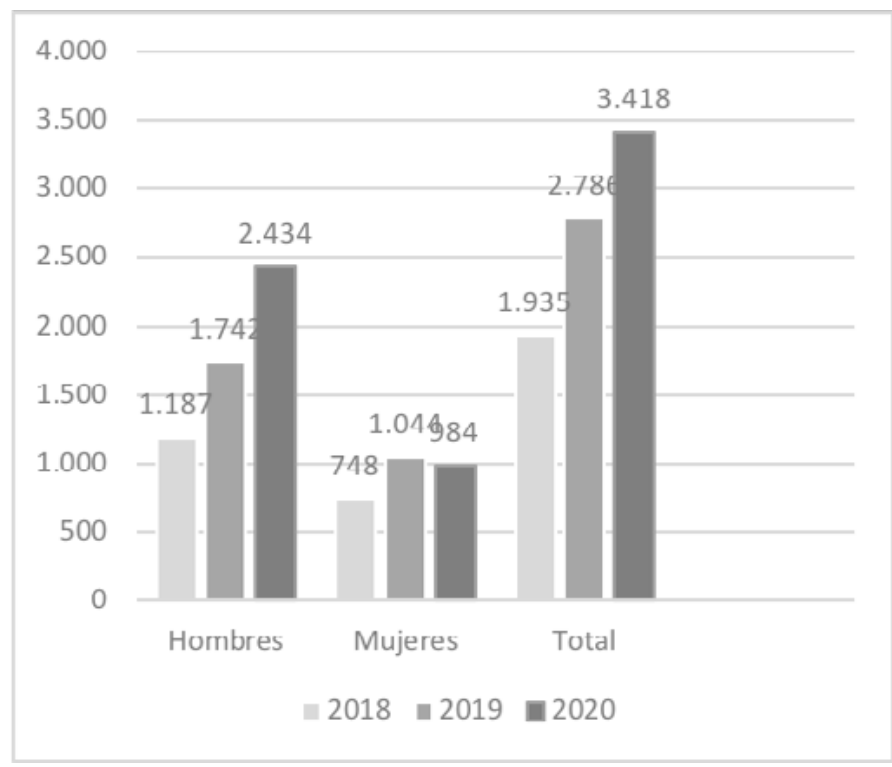

Fuente: Instituto Nacional de Estadísticas y Censos (2021)

En relación al personal agrícola permanente contratado por las empresas e instituciones agrícolas, se evidencia que el comportamiento en dicho sector laboral se ha mantenido estable, a pesar de la adversidad de varios eventos como la pandemia Covid-19, en el año 2020 se registró un total de tres mil cuatrocientos diez y ocho trabajadores en relación a los dos mil setecientos ochenta y seis del año 2019, y; mil novecientos treinta y cinco trabajadores entre hombres y mujeres del 2018 en el sector agrícola en la provincia de Tungurahua.

\section{Figura 11}

Trabajadores remunerados ocasionales

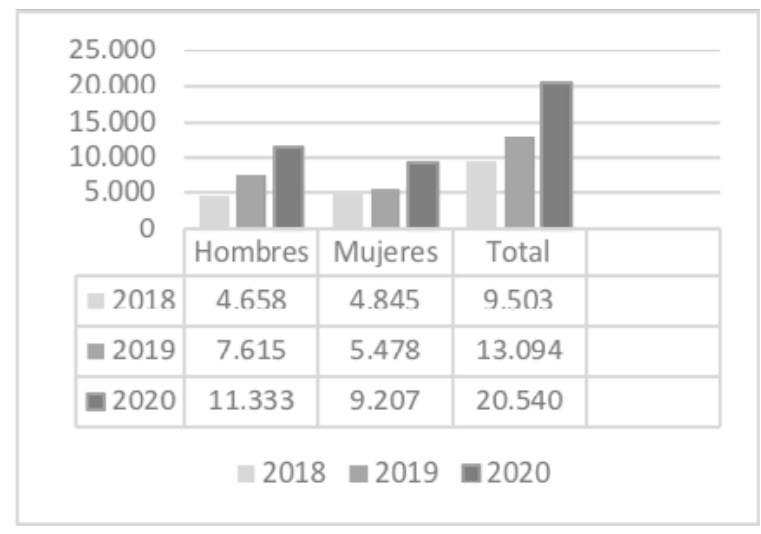

Fuente: Instituto Nacional de Estadísticas y Censos (2021) 
El sector agrícola en la provincia de Tungurahua constituye uno de los más importantes que abastece el consumo nacional, de tal manera se evidencia una participación estable en los trabajadores remunerados ocasionales del sector frente a los varios eventos adversos como el confinamiento e incendios forestales presentados en el 2020, y que se contabilizan un total de veinte mil quinientas cuarenta personas para dicho año en relación a las trece mil noventa y cuatro del año 2019.

Figura 12

Tasa trimestral de participación en la PEA de la ciudad de Ambato

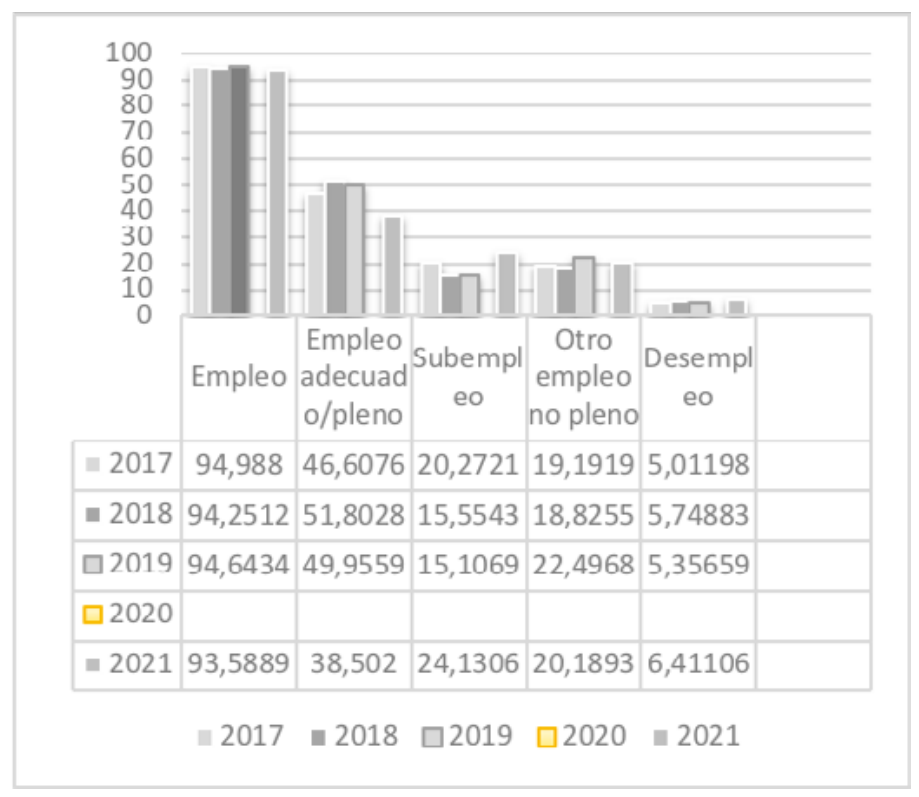

Fuente: Banco Central del Ecuador (2021)

El indicador de coyuntura del mercado laboral en la ciudad de Ambato, presenta un incremento paulatino año tras año de la tasa de desempleo, evidenciándose para el primer trimestre del año 2021 el $6,41 \%$. El subempleo en el mismo año registra un $24,13 \%$, de igual manera la categoría de otro empleo no pleno con el 20,19\%. La situación del confinamiento de la pandemia contribuyó notablemente a que aumente la cantidad de personas que se quedaron sin trabajo, se tuvo que reducir la plantilla de trabajadores. 


\section{Figura 13}

Porcentaje de turistas

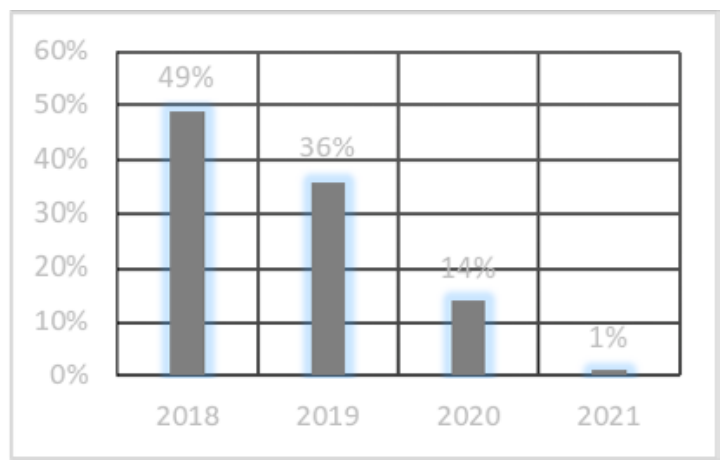

Fuente: Observatorio Económico y Social de Tungurahua (2019)

El porcentaje de los turistas que ingresaron a la provincia de Tungurahua durante el año 2018 fue del 49\%, a partir del 2019 se registró un decremento del 13\% en el turismo. En el año 2020 el flujo de personas que ingresaron a los distintos lugares turísticos es del $14 \%$. Una vez declarada la emergencia sanitaria en el país, el turismo se cerró por disposición del Gobierno central debido a la crisis mundial del Covid 19 que afectó las distintas actividades productivas. Para el año 2021 se contabiliza el $1 \%$ de turistas que han ingresado a la provincia desde el mes de enero hasta junio.

\section{Figura 14}

Participación de los sectores económicos

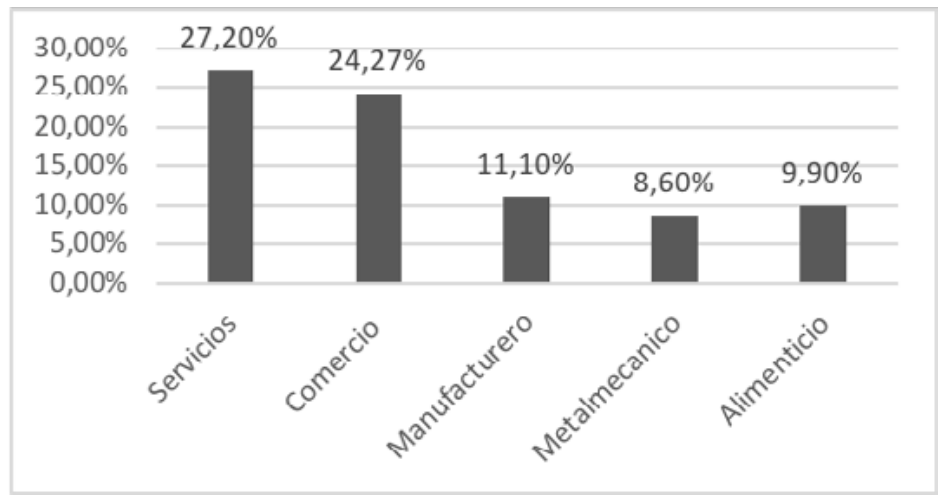

Fuente: Cámara de Comercio de Ambato (2021)

El sector económico que prevalece durante la crisis sanitaria es el de servicios con el $27.2 \%$, seguido de la actividad comercial con el $24.7 \%$, en el tercer lugar se destaca los sectores manufactureros como es el cuero y calzado con el $11.1 \%$, con el $9.9 \%$ representa a los sectores 
alimenticios y metalmecánico, mientras que el $8.6 \%$ que corresponde al sector textil y otras industrias como madereros y agrícolas son los más afectados por la pandemia.

Figura 15

Participación laboral de las empresas

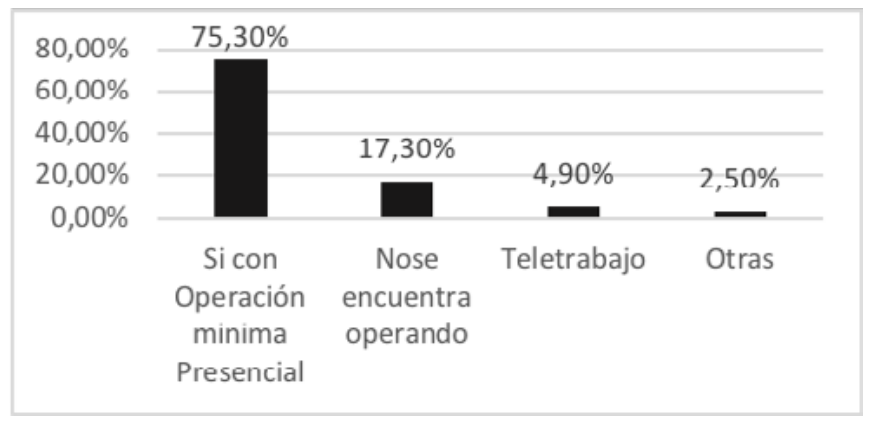

Fuente: Cámara de Comercio de Ambato (2021)

El $75.3 \%$ de las organizaciones empresariales de los diferentes sectores económicos en Tungurahua se han encontrado con operación mínima de forma presencial más teletrabajo, mientras que el $17.3 \%$ de los negocios no se encuentran operando, el $4.9 \%$ está laborando en forma de teletrabajo, mientras que el $2.5 \%$ están operando de varias maneras tales como la reducción de la jornada laboral, trabajos en horarios extendidos, trabajando de forma completa lo cual ha afectado al sector empresarial, ya que las actividades comerciales no se han generado de forma estable.

\section{Figura 16}

Ingresos por ventas en el transcurso de la pandemia

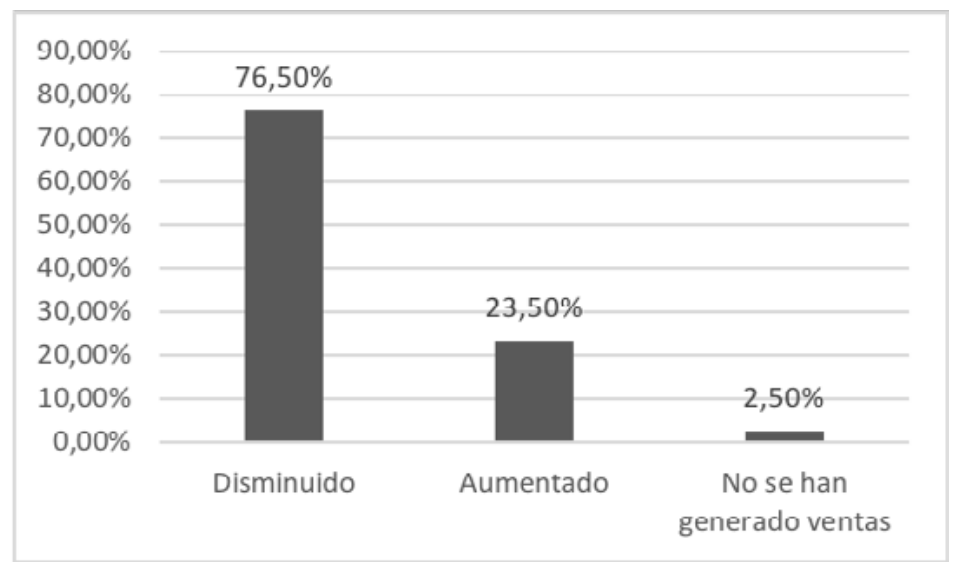

Fuente: Cámara de Comercio de Ambato (2021)

En el transcurso de la crisis por la emergencia sanitaria, los ingresos económicos han disminuido en el $76.5 \%$, específicamente en las empresas que se dedican a las actividades comerciales como 
son los negocios de venta de ropa y calzado, de igual manera los restaurantes, hoteles y demás servicios han sido afectados en sus ventas. Mientras que en algunos establecimientos comerciales no se ha aumentado las ventas reflejadas en el $23,5 \%$. No se han generado ventas en un $2,5 \%$ durante el transcurso de la pandemia.

Figura 17

Porcentajes representativos en las ventas comerciales

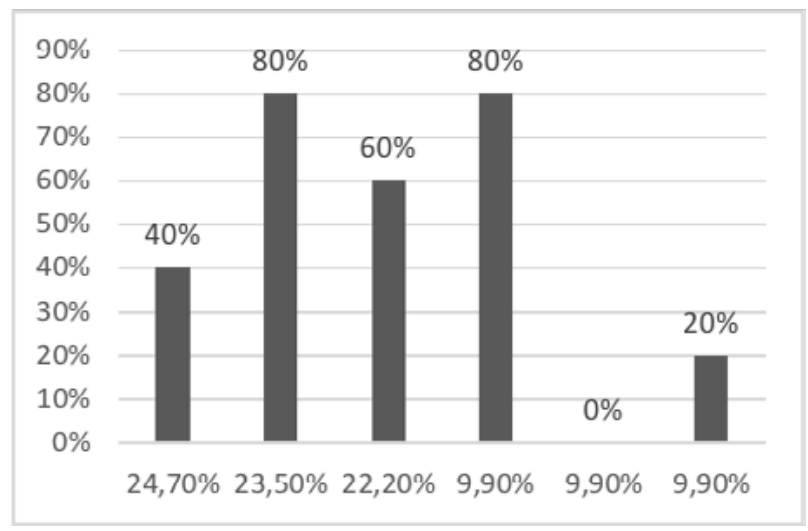

Fuente: Cámara de Comercio de Ambato (2021)

El $24,7 \%$ de los propietarios de negocios tuvieron impactos desfavorables en las ventas de sus productos en un $40 \%$, el $23,5 \%$ de los negocios han sido afectados en un $80 \%$, el $22,2 \%$ de las actividades comerciales han visto reducidos sus ingresos al $60 \%$, el 9,9\% de los establecimientos se ha producido un decremento de más del $80 \%$ en sus ventas. El $9,9 \%$ de las actividades productivas han disminuido sus ventas en el $20 \%$ y $0 \%$ respectivamente.

Figura 18

Decisiones adoptadas para reactivar los negocios

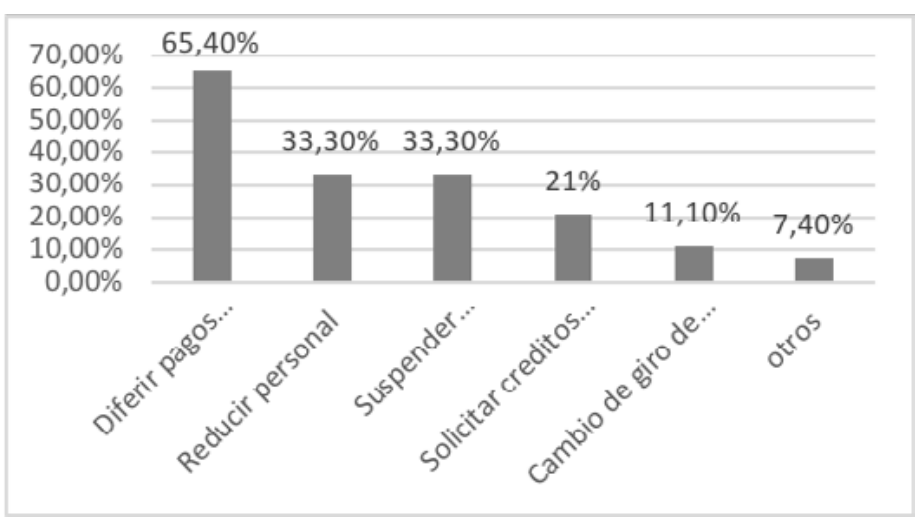

Fuente: Cámara de Comercio de Ambato (2021) 
Las decisiones adoptadas por los empresarios ante la difícil situación que ha generado la emergencia sanitaria para continuar sus labores y no cerrar sus establecimientos, se registra un $65.4 \%$ de los negocios que representan los diferentes sectores económicos, han diferido pagos con proveedores y obligaciones, el 33.3\% de los negocios hicieron una reducción del personal y de igual manera se suspendió temporalmente a empleados , el $11.1 \%$ han optado por dar un giro de negocio y el $7.4 \%$ eligieron varias decisiones entre ellas la implementación de ventas novedosas por Internet, han buscado y reestructurado estrategias de comercialización y atención al cliente, además está la elaboración de productos económicos y con precios accesibles.

Figura 19

Acciones realizadas ante suspensión temporal de los negocios

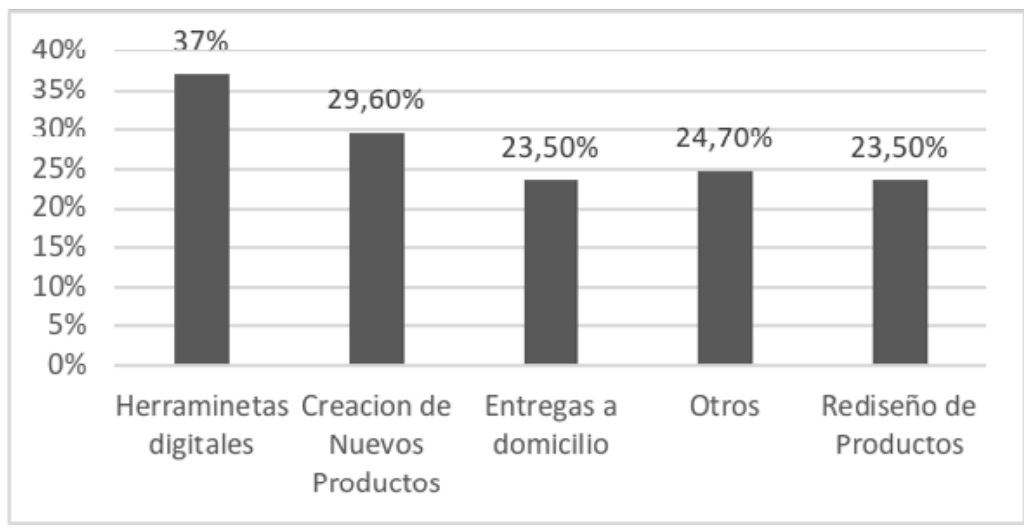

Fuente: Cámara de Comercio de Ambato (2021)

Las empresas han tenido que reinventarse o aplicar nuevas acciones o estrategias para activar sus operaciones, el $37 \%$ de las organizaciones han tenido que optar por aplicar herramientas digitales, el $29.6 \%$ de los negocios han implementado la creación de nuevos productos para la venta, el $23.5 \%$ han tomado la opción de rediseñar sus productos y vender a domicilio, mientras que el $24.7 \%$ establecieron un manual de bioseguridad para continuar laborando, en especial los trabajos o los servicios de campo; es decir, que deben movilizarse a diferentes lugares para cumplir sus labores, también liquidar activos que ya están fuera de uso para aumentar sus ingresos y poder mantener activos sus negocios entre otras acciones. 


\section{Figura 20}

Incremento en el presupuesto por implementación de la bioseguridad

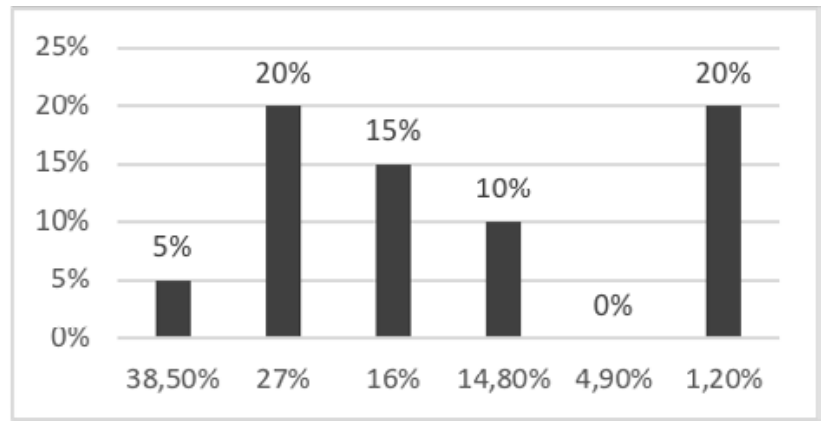

Fuente: Cámara de Comercio de Ambato (2021)

Las empresas para seguir operando y cumplir con los requisitos de funcionamiento establecidos por la ordenanza pública han tenido que implementar varios protocolos de bioseguridad, mismos que representan desembolsos considerables en su presupuesto. El 35.8\% de las empresas consideró un $5 \%$ para la ejecución de su plan de contingencia, el $27.2 \%$ de las organizaciones incrementó un $20 \%$ en sus gastos para brindar seguridad sanitaria, el $16 \%$ de los negocios destinó un $15 \%$ del presupuesto, $14,8 \%$ de los empresarios participaron con el $10 \%$ en programas de prevención del Covid, el 1,2\% de las empresas incrementaron sus gastos en más del $20 \%$ en protocolos de bioseguridad, mientras que el 4,9\% de los negocios no implementaron ningún programa de protección sanitaria.

\section{Conclusiones}

Un evento adverso cualquiera que sea su denominación como la actividad volcánica, deslizamientos de tierra, inundaciones, incendios forestales, sismos, la misma pandemia Covid-19; si bien obedecen a situaciones provenientes de la propia naturaleza, en su mayoría traen consigo consecuencias negativas como la pérdida de vidas humanas, económicas, ambientales, las cuales superan en mucho la capacidad de las personas organizadas en sociedad por sus propios medios, que puedan hacer frente a estos acontecimientos.

La Constitución de la República con su contenido normativo busca que tanto la ciudadanía, como las diferentes instituciones gubernamentales y técnicas, fortalezcan su capacidad para identificar posibles situaciones de riesgo, cada una dentro de sus respectivos ámbitos, además de llevar a cabo la información de los mismos incorporando las medidas que se estimen oportunas para reducirlos y en caso de ocurrencia minimizar sus consecuencias.

El constante advenimiento de eventos adversos ha hecho que el Estado y los distintos entes gubernamentales se preocupen en formular planes, política, programas y proyectos, tendientes a hacer frente a crisis, emergencias, desastres o catástrofes, con el propósito de mitigar los efectos negativos que estos pudieren generar; no obstante, el esfuerzo resulta mermando sin el aporte económico necesario y el compromiso serio de todos los actores sociales. 


\section{Referencias}

Asamblea Nacional del Ecuador. (2014). Código Orgánico Integral Penal. Registro Oficial. Última modificación: 17-feb.-2021. https://www.defensa.gob.ec/wp-content/uploads/downloads/2021/03/COIP act feb-2021. pdf

Asamblea Nacional del Ecuador. (2008). Constitución de la República del Ecuador. Sección Novena. Gestión del Riesgo, Art. 389, numeral 4, 175. http://www.asambleanacional.gob.ec/sites/default/files/documents/ old/constitucion de bolsillo.pdf

Asamblea Nacional del Ecuador. (2013). Proyecto de Ley del Sistema Nacional Descentralizado de Gestión de Riesgos.

Banco Central del Ecuador. (2021). Indicadores de coyuntura del mercado laboral ecuatoriano. https://contenido.bce.fin.ec/documentos/PublicacionesNotas/Catalogo/IEMensual/m2031/IEM-427a-e.xlsx

Cámara de Comercio de Ambato. (2021). Departamento de Marketing y Comercialización. Información proporcionada mediante entrevista a la Ing. Sandra Milena Salazar Directora del Departamento de Marketing y Comercialización de la CCA.

Gaibor, A., \& Pilco, J. (2021). Resiliencia frente a las amenazas naturales en la parroquia San Lorenzo, cantón Guaranda, provincia Bolívar. [Tesis de grado, Universidad Estatal de Bolívar].

Hernández, A. (2020). Evaluación y Ejecución de Proyectos de Seguimiento Escolar. EVIN 400. Universidad de Panamá.

Instituto Nacional de Estadísticas y Censos. (2019). Encuesta de Superficie y Producción Agropecuaria Continua (ESPAC) 2018. INEC

https://docs.google.com/spreadsheets/d/1p NXjQ1MjUKNNKhgFLkfxHym3N1UU3 H/edit?usp=sharing\&oui$\underline{d=109716016797598027196 \& r t p o f=t r u e \& s d=t r u e}$

Instituto Nacional de Estadísticas y Censos. (2020). Encuesta de superficie y producción agropecuaria continua (ESPAC) 2019. INEC https://docs.google.com/spreadsheets/d/1l1or9S8BrLQ4alce3EAAcd33jkiTVwNA/ edit?usp=sharing\&ouid $=109716016797598027196 \&$ rtpof $=$ true $\&$ sd $=$ true

Instituto Nacional de Estadísticas y Censos. (2021). Encuesta de superficie y producción agropecuaria continua (ESPAC) 2020. INEC https://docs.google.com/spreadsheets/d/1GRsE1e9TSAQOYrBAXDswCI5 AWDQftvS/edit?usp=sharing\&ouid $=109716016797598027196 \&$ rtpof=true $\&$ sd=true 
Instituto Nacional de Estadísticas y Censos. (2021). Registro Estadístico de Defunciones Generales. Boletín Técnico. INEC. https://www.ecuadorencifras.gob.ec/documentos/web-inec/Poblacion y Demografia/Defunciones Generales 2020/boletin tecnico edg 2020 v1.pdf

Instituto Nacional de Estadísticas y Censos. (2021). Encuesta Nacional de Empleo, Desempleo, y Subempleo. INEC. https://www.ecuadorencifras.gob.ec/empleo-julio-2021/

Maguiña Vargas, C., Gastelo Acosta, R., \& Tequen Bernilla, A. (2020). El nuevo Coronavirus y la pandemia del Covid-19. Revista Médica Herediana, 31(2), 125-131.

Mendoza, D. (2019). Condiciones ambientales y vulnerabilidad ante eventos adversos en el barrio Aire Libre de la Ciudad de Esmeraldas. [Tesis de grado, Pontificia Universidad Católica del Ecuador]. Repositorio digital PUCESE. https://repositorio.pucese.edu.ec/handle/123456789/1923

Moncayo Theurer, M., Velasco, G., Mora, C., Montenegro, M., \& Cordova, J. (2017). Terremotos mayores a 6.5 en escala Richter ocurridos en Ecuador desde 1900 hasta 1970. Ingeniería, 21(2), 55-64.

Observatorio Económico y Social de Tungurahua. (2019). Sector turístico Ecuador: alojamiento y servicios de comida https://blogs.cedia.org.ec/obest/wp-content/uploads/sites/7/2020/06/Turismo-en-Ecuador-Alojamiento-y-servicios-de-comida.pdf

Paredes Paz, M., \& Sánchez Paredes, D. (2020). Propuesta de un Plan de Restauración en Áreas afectadas por Incendios de Origen Antrópico en Los Páramos del Parque Nacional Llanganates del Cantón Tungurahua. [Tesis de grado, Universidad Estatal Amazónica]. Repositorio UEA. https://repositorio.uea.edu.ec/ handle/123456789/842

Servicio Nacional de Gestión de Riesgos y Emergencias. (2020). Informe de Situación - Incendios Forestales https://www.gestionderiesgos.gob.ec/wp-content/uploads/2020/11/Informe-de-Situacion-No-005-Incendios-Forestales-08112020 17h00.pdf

UNESCO. (2014). Gestión del riesgo de desastres para el patrimonio mundial. https://portals.iucn.org/library/ sites/library/files/documents/2010-105-Es.pdf

Ulloa, F. (2011). Manual de gestión del riesgo de desastre para comunicadores sociales. UNESCO. http://repo. floodalliance.net/jspui/handle/44111/2151

Villavicencio Silva, V. (2019). Mecanismo de respuesta del ejército ecuatoriano frente a eventos adversos en el territorio nacional (Unidad de Respuesta Inmediata URI). [Tesis de grado, Instituto de Altos Estudios Nacionales]. Repositorio IAEN.

Yánez Lucero, J. (2017). Capacidad de respuesta del personal de emergencia del Hospital San Francisco de Quito del Instituto Ecuatoriano de Seguridad Social frente a un evento adverso de víctimas en masa me- 
diante la ejecución de simulacros en el período febrero del 2017 - mayo del 2017. [Tesis de grado, Pontificia Universidad Católica Del Ecuador]. Repositorio PUCE. http://repositorio.puce.edu.ec/handle/22000/13302 
Copyright (c) 2021 Jorge Oswaldo Tamayo Viera, Vicente Leonardo Pérez Yauli y Edison Manuel Arroba Freire

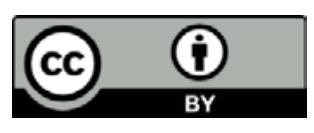

Este texto está protegido bajo una licencia internacional Creative Commons 4.0.

Usted es libre para Compartir-copiar y redistribuir el material en cualquier medio o formato - y Adaptar el documento - remezclar, transformar y crear a partir del material-para cualquier propósito, incluso para fines comerciales, siempre que cumpla las condiciones de Atribución. Usted debe dar crédito a la obra original de manera adecuada, proporcionar un enlace a la licencia, e indicar si se han realizado cambios. Puede hacerlo en cualquier forma razonable, pero no de forma tal que sugiera que tiene el apoyo del licenciante o lo recibe por el uso que hace de la obra.

Resumen de licencia - Texto completo de la licencia 